\title{
Temporary Anchorage Device Stability: Monocortical Versus Bicortical Anchorage Technique
}

\author{
Fateen Nur Ain Mohd Azmi ,Lim Shuang Ying, Wael Ahmed Bayomy Mohamed, Rozita Hassan* \\ Orthodontic Unit, School of Dental Sciences, Universiti Sains Malaysia, Kubang Kerian, Kelantan, 16150, Malaysia \\ *E-mail: rozitakb@usm.my
}

\begin{abstract}
Objective: To compare the stability of splinted and non-splinted miniscrew between monocortical and bicortical anchorage techniques.

Methods: Thirty-Six AbsoAnchor orthodontic miniscrews $(10 \mathrm{~mm} \times 1.5 \mathrm{~mm})$ were divided into four groups; nonsplinted monocortical, non-splinted bicortical, splinted monocortical and splinted bicortical anchorage techniques. The miniscrews were inserted into sectioned bovine bone blocks and the pull-out strength for each group of miniscrews was measured using Instron 8874; a crosshead speed of $2 \mathrm{~mm} /$ minute was applied. Six readings were taken for each group and the mean reading for each group was obtained. The data was analyzed using one-way ANOVA test with $95 \%$ confidence interval $(\mathrm{P}<0.05)$.

Results: The pull-out strength mean of splinted group was observed to be statistically higher than non-splinted. The mean of the pull-out strength of bicortical was higher than monocortical in both splinted and non-splinted groups, however it is not significant.

Conclusion: Both splinted monocortical and bicortical anchorage miniscrews were significantly stable than nonsplinted counterpart. However, in monocortical and bicortical anchorage, there was no significant difference of the pullout strength between splinted and non-splinted miniscrews.
\end{abstract}

Keywords: orthodontic, bicortical, miniscrew, monocortical, splint

\section{Introduction}

Orthodontic anchorage is used widely in many orthodontic clinical situations. Among the types of temporary anchorage devices used in orthodontics are osseointegrated implants, miniplates system and miniscrews. ${ }^{1}$ Osseointegrated implants were modified from dental implants. They provide maximum anchorage for large and difficult tooth movement. However, their insertion method is complex and they are bigger in size. Miniscrews however, are developed from the screws of maxillofacial plating system. ${ }^{1}$ Their main advantage is that they are small in size. Therefore, even though osseointegrated implants provide good anchorage in the oral cavity, due to the size, temporary anchorage devices or miniscrews more preferable in some orthodontic cases. $^{2-3}$

Miniscrews in orthodontics are also well known to the smooth in surface, cheaper and reduce chair-side time insertion. ${ }^{4}$ With the availability of the advantages mentioned, miniscrews are easy to use and can be placed just under local anesthesia hence giving the possibility of immediate loading. Unlike dental implants, which acquire their stability via osseointegration, miniscrews obtain maximum stability mechanically via primary retention..$^{5-6}$ Additionally, miniscrews can provide stationary anchorages for various tooth movements and even make it possible to move the tooth in directions that cannot be done with traditional osseointegrated implants anchorage. Even without patient's compliance, the miniscrews can also be a success. ${ }^{7}$ Therefore, miniscrews approach in orthodontics nowadays is getting popular.

As miniscrews are small, its handling should be professional when it is being used in either clinical or for research purposes. It has been shown that miniscrew biomechanical stability is greater when placed perpendicular to the cortical bone. Placement angles of less than $60^{\circ}$ will reduce the biomechanical stability of the miniscrews when orthopedic forces are applied in different directions. It is also stated that cortical bone thickness of more than $1 \mathrm{~mm}$ will increase the success rate of the miniscrews than cortical bone with $0.5 \mathrm{~mm}$ thickness8. This is related to a new technique called 'bicortical anchorage technique' which is introduced to increase the cortical bone thickness. This technique takes 
anchorage from both buccal and lingual cortical bone plate of the jaw bones. ${ }^{6}$

Other than that, regarding the miniscrews resistance to being pull out, the factors involved are the type of material, device diameter, length of thread, and shear strength of the material into which the screw is inserted. ${ }^{9}$ These things should also be considered when conducting the research in order to get more accurate results. Some studies state that the pull-out strength of the miniscrews from its site of implantation is a fundamental parameter to measure its primary retention which is also linked to bone density, volume, and cortical thickness. ${ }^{10-11}$

With considerations of all the facts, hence, in this research, we were comparing monocortical versus bicortical anchorage technique in increasing the stability of the orthodontic miniscrews whether the miniscrews is splinted or not, through measuring of the pull-out strength of the miniscrews.

\section{Methods}

\section{Study design and methodology}

This was an in-vitro experimental study on the pull-out strength of miniscrews using the different anchorage techniques applied on bovine bone blocks. The four different anchorage techniques in this study were nonsplinted monocortical (NSM), non-splinted bicortical (NSB), splinted monocortical (SM) and splinted bicortical (SB) anchorage technique. Thirty six AbsoAnchor orthodontic miniscrews $(10 \mathrm{~mm} \times 1.5 \mathrm{~mm})$ were screwed into sectioned bovine bone blocks and the pull-out strength for each group of orthodontic miniscrews was measured using Instron 8874; a crosshead speed of $2 \mathrm{~mm} /$ minute was applied. The study protocol was reviewed and approved by the Human Research Ethics Committee of USM (USM/ JEPeM/ 15060205)

The cortical and cancellous bone blocks were delivered from the tissue bank of School of Medical Sciences, Universiti Sains Malaysia. These bone blocks were sectioned to similar size measuring $(20 \mathrm{~mm} \times 7 \mathrm{~mm} \times$ $7 \mathrm{~mm}$ ) using laboratory stone discs in order for the bone blocks to fit into the Instron 8874. The cortical bone thickness was made constant to $1 \mathrm{~mm}$ each. The bone blocks were stored in the 'cold room' under a temperature of $6^{\circ} \mathrm{C}$ when not in used.

On the day of testing, the bovine bone blocks were thawed to room temperature. Surgical straight fissure bur was used to drill a hole through the whole length of the bovine bone blocks prior to miniscrews placement. A total of thirty-six (1.5mm x $10 \mathrm{~mm} /$ diameter $\times$ length) selfdrilling AbsoAnchor miniscrews were inserted into the 24 bovine bone blocks which were divided into four groups with different designs for each group: 6 monocortical, 6 bicortical, 12 splinted monocortical, 12 splinted bicortical. The miniscrews were inserted perpendicularly to the cortical bone surface. Two miniscrews for the splinted groups were inserted with $1 \mathrm{~cm}$ distance in between the miniscrews and are joined together using stainless steel wire of diameter $0.9 \mathrm{~mm}$ and orthodontic ligature wire. Figure 1 shows the miniscrew inserted using different anchorage technique in the bovine bone blocks

After that, cold cured acrylic was added at both ends of the bovine bone blocks for extra strength to hold the cortical and cancellous bone layers together. Completed samples were then inserted into the testing machine (Instron 8874) at Material Testing Laboratory in School of Dental Sciences Universiti Sains Malaysia (Figure 2). Then, the pull-out strength (Quantification of the tensile forces required to pull a screw out) was measured at a speed of $2 \mathrm{~mm} / \mathrm{min}$ for all the groups. Six readings were taken for each group of the anchorage techniques and were recorded in the unit of Newton $(\mathrm{N})$. The procedures were performed by the same operator.

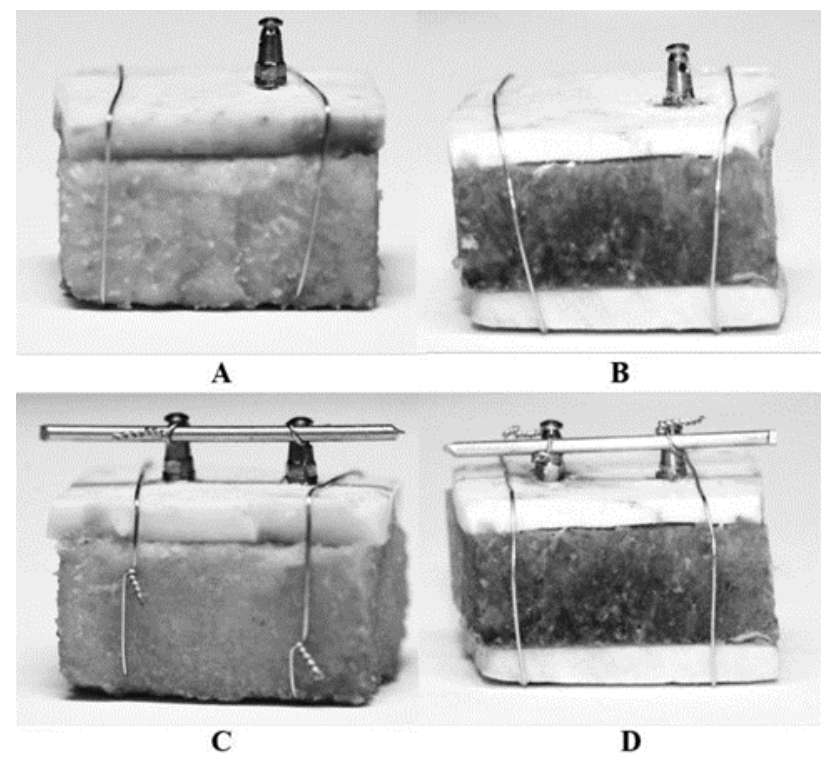

Figure 1. Four different anchorage techniques and the insertion of miniscrews in fresh bovine bone blocks. A, Non-splinted monocortical anchorage technique, B, Non-splinted bicortical anchorage technique, C, Splinted monocortical anchorage technique. D, Splinted bicortical anchorage technique.

Table 1. Means, standard deviations (SD), standard errors (SE) and minimum and maximum values of pull-out test results

\begin{tabular}{ccccccc}
\hline $\begin{array}{c}\text { Anchorage } \\
\text { technique }\end{array}$ & $n$ & $\begin{array}{c}\text { Mean } \\
(N)\end{array}$ & $S D$ & $S E$ & $\begin{array}{c}\text { Minimum } \\
(N)\end{array}$ & $\begin{array}{c}\text { Maximum } \\
(N)\end{array}$
\end{tabular}

Non-splinted

$\begin{array}{lllllll}\text { monocortical } & 6 & 155.1 & 45.8 & 18.7 & 111.6 & 222.4\end{array}$

(NSM)

$\begin{array}{ccccccc}\begin{array}{c}\text { Non-splinted } \\ \text { bicortical } \\ \text { (NSB) }\end{array} & 6 & 201.8 & 69.1 & 28.2 & 123.6 & 318.0\end{array}$




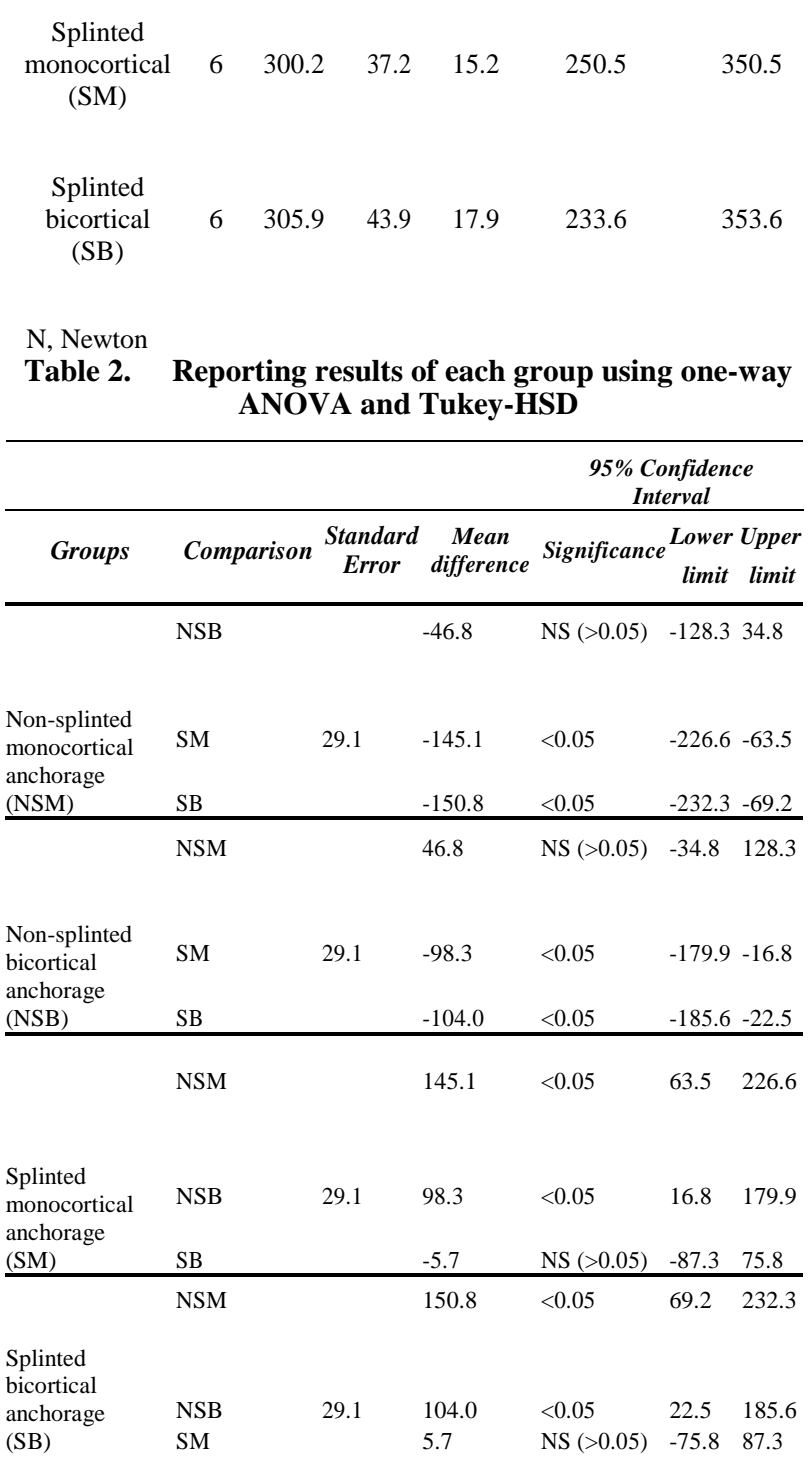

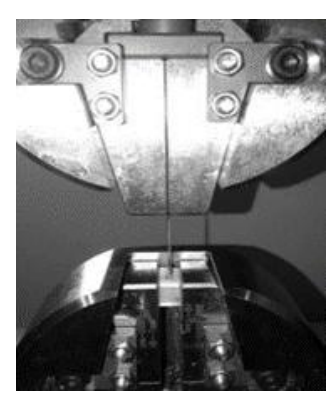

${ }^{\text {a. }}$ NS, Not significant

Figure 2. Bovine bone specimens being inserted into Instron 8874.

\section{Statistical analysis}

The pull-out strength was measured as the peak force needed to produce the first movement of the miniscrews in the bovine bone blocks. The data obtained for each of the category of the miniscrews were analyzed using SPSS (Windows, version 22.0) and the significance level is set at $5 \%$ (P-value < 0.05). The comparison of pull-out strength between non-splinted and splinted, monocortical and bicortical miniscrews were presented using One-way analysis of variance (ANOVA) and Tukey-HSD (honest significance difference) test.

\section{Results}

In this trial, a total of six readings were successfully recorded for each group and the mean reading for each group was obtained (Table 1). The mean results were compared between each group respectively using one-way analysis of variance (ANOVA) in order to obtain the significant differences in between all present groups (Table 2).

In terms of pull-out strength, the non-splinted monocortical (NSM) anchorage had the lowest mean pullout strength which was $155.08 \mathrm{~N}(\mathrm{SD}=45.8)$. In contrast, splinted bicortical anchorage (SB) showed maximum mean pull-out strength with a result of $305.87 \mathrm{~N}$ $(\mathrm{SD}=43.9)$ which is significantly higher compared to the NSM group. There was a trend of greater pull-out strength within the splinted anchorage groups (SM and SB) in comparison to the non-splinted anchorage groups (NSM and NSB).

The maximum mean pull-out strength was achieved following the sequence in decreasing order: $\mathrm{SB}>\mathrm{SM}>$ NSB $>$ NSM.

By using post-hoc multiple comparisons, P-values $(\mathrm{P}<0.05)$ showed significant difference in pull-out strength in all groups except for the comparison between non-splinted monocortical anchorage (NSM) and nonsplinted bicortical (NSB) which gave a higher p-value $(\mathrm{P}=0.399)$. Similarly, the contrast between splinted bicortical anchorage and splinted monocortical anchorage also shows non-significant difference $(\mathrm{P}=0.997)$. Similarly, the contrast between splinted bicortical anchorage (SB) and splinted monocortical anchorage (SM) also shows non-significant difference $(\mathrm{P}=0.997)$ but SB shows a slightly higher reading compared to SM.

\section{Discussion}

\section{Primary stability}

Skeletal anchorage of temporary anchorage device can be classified into osseointegration or mechanical interdigitation. $^{12-13}$ Primary stability of the orthodontic miniscrew occurs when mechanical stability is achieved in the bone immediately after the miniscrew is inserted into the bone. ${ }^{14-18}$

The primary stability of temporary skeletal anchorage can be influenced by many factors including geometry of miniscrew, bone characteristics, angulation placement of miniscrew and pullout force which is the major factor for clinical success with miniscrews. ${ }^{19-20}$ These factors may directly or indirectly affect the results. The thread shape of the miniscrew has been shown to give a significant resistance to pull-out strength. ${ }^{19,}{ }^{21}$ Therefore, the same type of miniscrew was used in all trials hence retaining the same geometry for each trial ensuring that the thread 
shape does not affect the results. The bone characteristics was also made constant during preparation as cortical thickness was also found to have influence the primary stability as the cortical bone has a higher Young's modulus which is able to resist more deformation and sustain higher loads compared to the cancellous bone. ${ }^{22}$ Therefore, all the possible variables that may affect the primary retention of the temporary anchorage device were made constant in order to increase the accuracy of the trials.

\section{Monocortical anchorage vs bicortical anchorage technique}

In this study, it has been shown that there is no significant statistical difference with respect to the bicortical anchorage technique compared to monocortical anchorage technique $(\mathrm{P}>0.05)$. In terms of the result, although the bicortical anchorage technique produced better resistance which gives higher pull-out force reading. However, the results were not significant enough thus making it incoherent with a previous report which hypothesized that the pull-out force for bicortical anchorage technique is significantly greater than monocortical anchorage technique6. It has also been mentioned in another report that placement of miniscrew to bicortical bone plates would make a significant difference in anchorage stability. The primary stability of miniscrews is believed to result from the placement of the miniscrew using monocortical or bicortical anchorage. ${ }^{23}$

Utilization of bicortical plates of the bovine bone provides mechanical interlock between the thread of the screw. By nature, cortical bone is higher in density than cancellous bone thus permits a high value of interconnection. These miniscrew are mechanically integrated, their anchorage value is influenced by the quality and quantity of the bone which they are placed into. ${ }^{3,23-27}$ However, another report states that it is mainly the thickness (quantity) and not the density (quality) of the cortical bone that guarantees the stability of miniscrews20. Higher miniscrew implant failure rates were observed with cortical bone thickness less than $1 \mathrm{~mm}$ and reduced prognosis has been seen in cases with cortical bone thickness less than $0.5 \mathrm{~mm} .^{18,24,27-}$ ${ }^{28}$ Hence, in cases where there is limited cortical bone thickness such as the cortical bone between the canine and first premolar on the buccal side of the mandible, ${ }^{29}$ bicortical anchorage technique can be advantageous.

\section{Splinted anchorage vs non-splinted anchorage technique}

To date, there is no study done to compare the maximum pull-out strength between monocortical and bicortical anchorage technique done using bovine bone to test the differences between splinted and non-splinted miniscrews. In this study, there were significant difference between the splinted and non-splinted group of both monocortical anchorage and bicortical anchorage technique $(\mathrm{P}<0.05)$. Miniscrew stability can be further enhanced by splinting two miniscrews together. ${ }^{30}$ Therefore, miniscrews were splinted together to minimize micromotion and encourage stability as the vector forces are distributed evenly between two miniscrews. This result shown by the increasing trend of the maximum pull-out force produced by bicortical anchorage technique, the results are in accordance to the previous hypothesis made before the trials were executed.

\section{Conclusions}

Both splinted monocortical and splinted bicortical anchorage miniscrews are stable and had higher pull-out strength than non-splinted counterpart and despite greater pull-out strength in bicortical anchorage in comparison to monocortical anchorage in either non-splinted or splinted miniscrews, the differences were not significant. The findings can be used as a guidance in conditions where there is inadequate cortical bone thickness and the abovementioned anchorage techniques can be taken into consideration.

\section{Acknowledgment}

We would like to thank the staff of Dental Technology Laboratory and Dental Materials Laboratory, School of Dental Sciences, Universiti Sains Malaysia for lending their helps during the preparation of bone blocks and the managing of Instron 8874 during the research.

\section{References}

1. Laura Mitchell. An Introduction to Orthodontics. Oxford University Press, Great Clarendon Street, Oxford OX2 6DP, United Kingdom; 2007.

2. Kanomi, R. Mini-implant for orthodontic anchorage. Journal of Clinical Orthodontics. 1997; 31,763-767.

3. Miyawaki S, Koyama I, Inoue M, Mishima K, Sugahara T, Takano-Yamamoto T. Factors associated with the stability of titanium screws placed in the posterior region for orthodontic anchorage. American Journal of Orthodontics and Dentofacial Orthopedics. 2003;124,373-378.

4. Fritz U, Ehmer A, Diedrich P. Clinical suitability of titanium microscrews for orthodontic anchoragepreliminary experiences. Journal of Orofacial Orthopedics. 2004;65,410-418.

5. Melsen B, Verna C. A rational approach to orthodontic anchorage. Progress in Orthodontics. 1999;1,10-22.

6. Brettin BT, Grosland NM., Qian F, Southard KA, Stuntz TD, Morgan TA, Marshall SD, Southard TE. Bicortical vs monocortical orthodontic skeletal anchorage. American Journal of Orthodontics and Dentofacial Orthopedics. 2008; $134,625-635$

7. Kuroda S, Tanaka E. Risks and complications of miniscrew anchorage in clinical orthodontics. Japanese Dental Science Review. 2014;50,79-85.

8. Lee J, Kim JY, Choi YJ, Kim KH, Chung CJ. Effects of placement angle and direction of orthopedic force application on the stability of orthodontic miniscrews. Angle Orthodontist. 2013;83,667-673.

9. Pickard MB, Dechow P, Rossouw PE, Buschang PH Effects of miniscrew orientation on implant stability and resistance to failure. American Journal of Orthodontics and Dentofacial Orthopedics. 2010;137,91-99.

10. Choi JH, Park CH, Yi SW, Lim HJ, Hwang HS. Bone density measurement in interdental areas with simulated placement of orthodontic miniscrew implants. American Journal of Orthodontics and Dentofacial Orthopedics. 2009; 136,766-767.

11. Wang Z, Zhao Z, Xue J, Song J, Deng F, Yang P. Pullout strength of miniscrews placed in anterior mandibles of adult and adolescent dogs: a microcomputed tomographic 
analysis. American Journal of Orthodontics and Dentofacial Orthopedics. 2010;137,100-107.

12. Jason BC. Temporary anchorage devices in orthodontics: a paradigm shift. Seminars in Orthodontic. 2005;11,3-9.

13. Cochran DL, Schenk RK, Lussi A, Higginbottom FL, Buser D. Bone response to unloaded and loaded titanium implants with sandblasted and acid-etched surface: a histometric study in the canine mandible. Journal of Biomedical Materials Research. 1998;40,1-11.

14. Park HS, Jeong SH, Kwon OW. Factors affecting the clinical success of screw implants used as orthodontic anchorage. American Journal of Orthodontics and Dentofacial Orthopedics. 2006;130,18-25.

15. Moon CH, Lee DG, Lee HS, Im JS, Baek SH. Factors associated with the success rate of orthodontic miniscrews placed in the upper and lower posterior buccal region. Angle Orthodontist. 2008;78,101-106.

16. Wilmes B, Rademacher C, Olthoff G, Drescher D. Parameters affecting primary stability of orthodontic miniimplants. Journal of Orofacial Orthopedics. 2006;22,567570.

17. Song YY, Cha JY, Hwang CJ. Mechanical characteristics of various orthodontic mini-screws in relation to artificial cortical bone thickness. Angle Orthodontist. 2007;77,979985.

18. Mariana M, Margareth M, Monica T, Lincoln IN. Matilde $\mathrm{N}$. Is miniscrew primary stability influenced by bone density? Brazilian Oral Research. 2011;25,427-432.

19. Migliorati M, Signori A, Silvestrini Biavati A. Temporary anchorage device stability: an evaluation of thread shape factor. European Journal of Orthodontics. 2012;34,582-586.

20. Migliorati M, Benedicenti S, Signori A, Drago S, Barberis F, Tournier H, Silvestrini Biavati A. Miniscrew design and bone characteristics: An experimental study of primary stability. American Journal of Orthodontics and Dentofacial Orthopedics. 2012;142,228-234.

21. Gracco A, Giagnorio C, Parenti SI, Bonetti GA, Siciliani G. Effects of thread shape on pullout strength of miniscrews.
American Journal of Orthodontics and Dentofacial Orthopedics. 2012;142,187-190.

22. Liu TC, Chang CH, Wong TY, Liu JK. Finite element analysis of miniscrew implants used for orthodontic anchorage. American Journal of Orthodontics and Dentofacial Orthopedics. 2012;141,469-476.

23. Huja SS, Litsky AS, Beck FM, Johnson KA, Larsen PE. Pull-out strength of monocortical screws placed in the maxillae and mandibles of dogs. American Journal of Orthodontontics and Dentofacial Orthopedics. 2005; 127:307-313.

24. Melsen B. Mini-implants: where are we? Journal of Clinical Orthodontics. 2005;39,539-547.

25. Morarend C, Qian F, Marshall SD, Southard KA, Grosland NM, Morgan TA, McManus M, Southard TE. Effect of screw diameter on orthodontic skeletal anchorage. American Journal of Orthodontics and Dentofacial Orthopedics. 2009;136,224-229.

26. Wilmes B, Rademacher C, Olthoff G, Drescher D. Parameters affecting primary stability of orthodontic miniimplants. Journal of Orofacial Orthopedics. 2006;67,162 174.

27. Motoyoshi M, Yoshida T, Ono A, Shimizu N. Effect of cortical bone thickness and implant placement torque on stability of orthodontic mini-implants. International Journal of Oral Maxillofacial Implants. 2007;22,779-784.

28. Papageorgiou SN, Zogakis IP, Papadopoulos MA. Failure rates and associated risk factors or orthodontic miniscrew implants: A meta-analysis. American Journal of Orthodontics and Dentofacial Orthopedics. 2012;142,577595.

29. Kim JH, Park YC. Evaluation of mandibular cortical bone thickness for placement of temporary anchorage devices (TADs). Korean J Orthod. Jun 2012; 42(3): 110-117.

30. Lee JS, Kim DH, Park YC, Kyung SH, Kim TK. The Efficient Use of Midpalatal Miniscrew. Angle Orthodontist. 2004;74,711-714. 